\title{
Utilizing self-organization systems for modeling and managing risk based on maintenance and repair in petrochemical industries
}

\begin{abstract}
Maintenance is essential to ensure safe operation of equipment in normal conditions. Therefore, managers must identify the relative priorities and equipment maintenance requirements. Moreover, based on the results of equipment vulnerability assessments, maintenance programs can be developed and managed properly. There are different methods and techniques in the process of risk assessment and management and vulnerability of equipment. Seventy-six samples with different properties have been used in this study. Networks used in this study are self-organizing networks with constant weight, which include Kohonen networks. For this purpose, operation impact, operation flexibility, maintenance cost, impact of safety and environment and frequency parameters had been considered as input; and using this model, the risk level is calculated. Utilizing genetic algorithms, the structures of all self-organizing systems are optimized. In order to evaluate the accuracy of the model, we compare it with the fuzzy model, and the results indicate that self-organizing systems optimized with the genetic algorithm have higher ability, flexibility and accuracy than the fuzzy model in predicting risk.
\end{abstract}

Keyword: Self-organization feature maps; Genetic algorithms; Fuzzy model; Risk management; Petrochemical industry 